21st Particles and Nuclei International Conference (PANIC 2017)

International Journal of Modern Physics: Conference Series

Vol. 46 (2018) 1860036 (6 pages)

(C) The Author(s)

DOI: $10.1142 / \mathrm{S} 2010194518600364$

\title{
Breaking Symmetry Effects on Heavy Tetraquarks
}

\author{
J. Vijande \\ Unidad Mixta de Investigacin en Radiofsica e Instrumentacin Nuclear en Medicina (IRIMED), \\ Instituto de Investigacin Sanitaria La Fe (IIS- $\mathrm{La} \mathrm{Fe}$ ) \\ Universitat de Valencia (UV) and IFIC (UV-CSIC), Valencia, Spain \\ javier.vijande@uv.es
}

A. Valcarce

Departamento de Física Fundamental and IUFFyM, Universidad de Salamanca, E-37008 Salamanca, Spain valcarce@usal.es

J.-M. Richard

Université de Lyon, Institut de Physique Nucléaire de Lyon, IN2P3-CNRS-UCBL, 4 rue Enrico Fermi, 69622 Villeurbanne, France

j-m.richard@ipnl.in2p3.fr

Published 3 May 2018

In this contribution we present symmetry arguments that can be applied to study the stability of four-quark systems with two different masses. The role played by different symmetry breaking effects and the non-Abelian algebra of color forces is discussed in detail. In the particular case of hidden-flavor all-heavy four-quark states, $Q Q \bar{Q} \bar{Q}$, the system becomes unstable for standard color-additive models. Differences and similarities between $Q q \bar{Q} \bar{q}$ and $Q Q \bar{q} \bar{q}$ configurations are presented. In the latter case, its stability when the mass ratio $M / m$ increases was established almost forty years ago. In the former case, we find a kind of metastability between the lowest threshold, $(Q \bar{Q})-(q \bar{q})$ and the highest one, $(Q \bar{q})-(\bar{Q} q)$.

Keywords: Few-body systems; Tetraquarks.

\section{Introduction}

There are nowadays many discussions about the existence, structure, decay properties and production mechanisms of hadrons with a quark content richer than quarkantiquark or three-quark systems ${ }^{1,2}$. The ideas suggesting exotic hadrons were revisited with the discovery of the $X Y Z$ mesons and strengthened by the announcement

This is an Open Access article published by World Scientific Publishing Company. It is distributed under the terms of the Creative Commons Attribution 4.0 (CC-BY) License. Further distribution of this work is permitted, provided the original work is properly cited. 
of hidden-flavor pentaquarks ${ }^{3}$. The detection of a doubly-heavy baryon ${ }^{4}$ also opens the perspective of producing and identifying other hadrons with two units of heavy flavor.

In particular, the recent experimental findings have stimulated several studies dealing with multiquark states. In some recent papers, it is speculated about the existence of tetraquarks made of four-heavy constituents, $Q Q \bar{Q} \bar{Q}$. In some other papers, the authors rediscover the widely tackled sector of exotic tetraquarks with two units of flavor, $Q Q \bar{q} \bar{q}$, while their non-exotic partners, $Q q \bar{Q} \bar{q}$, have been much discussed in the context of the $X Y Z$ mesons.

In this contribution we discuss the stability patterns of four-quark states in close comparison to systems of charged particles, which have been well studied. We analyze the effects of symmetry breaking and color forces on the stability of four-body states.

\section{Model and formalism}

Building multiquark states involves hypotheses on how extrapolate from models that correctly accounts for the properties of ordinary mesons and baryons. We adopt here the simplest and most widely used option, which consists of two-body potentials with simple color dependence, including both a spin-independent (chromoelectric) and a spin-dependent (chromomagnetic) component. In the limit of very heavy quarks, the chromomagnetic forces vanish. It is thus interesting to consider the case of a purely chromoelectric interaction.

To compute the ground-state of a three-body baryon a crude variational approximation is often sufficient. For a tetraquark close to its threshold, this is drastically different. One has to estimate precisely $q_{1} q_{2} \bar{q}_{3} \bar{q}_{4}$ and its thresholds, to see whether a bound state exists. Moreover, the wave function has a $\left(q_{1} \bar{q}_{3}\right)\left(q_{2} \bar{q}_{4}\right)$ component and a $\left(q_{1} \bar{q}_{4}\right)\left(q_{2} \bar{q}_{3}\right)$ one, corresponding to its 'molecular' part, perhaps a $\left(q_{1} q_{2}\right)\left(\bar{q}_{3} \bar{q}_{4}\right)$ diquark-antidiquark component, and a collective component that prevails in the event of deep binding ${ }^{5}$. A similar situation is encountered in atomic physics: the deeply bound He atom, $\left(\alpha e^{-} e^{-}\right)$, is well described by a simple product of two functions. For the weakly bound $\mathrm{H}^{-},\left(p e^{-} e^{-}\right)$, one has to introduce a much more subtle wave function to demonstrate the stability against dissociation into $\mathrm{H}+e^{-6}$.

\section{General results based on symmetry breaking}

The analogy between the stability of few-charge systems and multiquarks in additive chromoelectric potentials offers a good guidance for identifying the favorable configurations. There are, however, some differences, mainly due to the color algebra replacing the simpler algebra of electric charges.

In Refs. 7, 8 it is explained why, unlike in the case of the positronium molecule, the equal-mass tetraquarks are unstable in the chromoelectric model with frozen color wave functions. In both the atom and quark cases, the four-body system and 
its threshold, after simple rescaling, are governed by a generic Hamiltonian

$$
H=\sum_{i} \frac{\vec{p}_{i}^{2}}{2 m_{i}}-\text { c.o.m. }+\sum_{i<j} g_{i j} v\left(r_{i j}\right) . \quad \sum_{i<j} g_{i j}=2 .
$$

In quantum mechanics, adding an antisymmetric term to a symmetric Hamiltonian lowers its minimum. If the symmetry is charge conjugation, $C$, the hydrogen molecule $M^{+} M^{+} m^{-} m^{-}$can be viewed as a deformed version of a positronium-like molecule. Its Hamiltonian can be written

$$
\begin{aligned}
H=\frac{\vec{p}_{1}^{2}}{2 M}+\frac{\vec{p}_{2}^{2}}{2 M} & +\frac{\vec{p}_{3}^{2}}{2 m}+\frac{\vec{p}_{4}^{2}}{2 m}+V=H_{0}+H_{1} \\
& =\left[\sum_{i} \frac{\vec{p}_{i}^{2}}{2 \mu}+V\right]+\left(\frac{1}{4 M}-\frac{1}{4 m}\right)\left(\vec{p}_{1}^{2}+\vec{p}_{2}^{2}-\vec{p}_{3}^{2}-\vec{p}_{4}^{2}\right)
\end{aligned}
$$

where $2 \mu^{-1}=M^{-1}+m^{-1}$. The $C$-parity breaking term, $H_{1}$, lowers the ground state energy of $H$ with respect to the $C$-parity even part, $H_{0}$, which is simply a rescaled version of the Hamiltonian of the positronium molecule. Since $H_{0}$ and $H$ have the same threshold, and since the positronium molecule is stable, the hydrogen molecule is even more stable, and stability improves when $M / m$ increases. Clearly, the Coulomb character of $V$ hardly matters in this reasoning. The key property is that the potential does not change when the masses are modified.

One can use the same reasoning to study the stability of four-charged particles when $C$-parity is preserved but particle symmetry is broken, in other words the $M^{+} m^{+} M^{-} m^{-}$configuration. The Hamiltonian is given by Eq. (2) replacing $\vec{p}_{2} \leftrightarrow$ $\vec{p}_{3}$. Using the same arguments as before one concludes that this configuration gains binding with respect to the threshold $\left(M^{+} m^{-}\right)-\left(M^{-} m^{+}\right)$that it shares with $H_{0}$. However, there is another threshold that lies lower, $\left(M^{+} M^{-}\right)-\left(m^{+} m^{-}\right)$. This threshold gains more from the symmetry breaking than the four-body molecule, and, indeed, it is found that the molecule becomes unstable for $M / m \gtrsim 2.2$. In other words, a protonium atom cannot polarize enough a positronium atom and stick to it. It remains that the hydrogen-antihydrogen system can form a kind of metastable molecule below the atom-antiatom threshold.

\section{4. $Q Q \bar{q} \bar{q}$ and $Q Q \bar{Q} \bar{Q}$ states}

The arguments we have explained above can be directly translated to four-quark systems: the $Q Q \bar{q} \bar{q}$ configuration becomes more and more bound when the mass ratio $M / m$ increases. This has been established in the early work of Ader, Richard and Taxil ${ }^{9}$, and discussed and confirmed in further studies. The critical value of $M / m$ for binding is somewhat model dependent. There is some kind of consensus that $b b \bar{q} \bar{q}$ is stable, with a binding energy that might reach $100 \mathrm{MeV}$ or more ${ }^{10}$. Arguments based on diquarks ${ }^{11}$ might considerably overestimate the binding ${ }^{12}$. Near the critical $M / m$, the explicit supplementation of meson-meson components in the wave function, and the account for chromomagnetic effects could be crucial ${ }^{13}$. The 
stability of $c c \bar{u} \bar{d}$ was first obtained by Janc and Rosina ${ }^{14}$ within an explicit quark model, that indeed, included a chromomagnetic term, and the result was confirmed in further works ${ }^{5,15}$.

A remaining problem is to understand why the positronium molecule lies slightly below its dissociation threshold, while a chromoelectric model associated with the color additive rule does not bind (at least according to most computations). This is due to a larger disorder in the color coefficients than in the electrostatic strength factors entering the Coulomb potential ${ }^{7}$. To illustrate this result let us analyze the consequence of asymmetries in the potential energy. Consider once more the Hamiltonian of (1), denoted $H[G]$, where $G=\left\{g_{i j}\right\}$. The simplest case is the symmetric set $G=G_{0}$ for which $g_{i j}=1 / 3, \forall i, j$. For the systems of interest, the set of coefficients is of the type $G=\left\{g^{\prime}, g^{\prime}, g^{\prime \prime}, g^{\prime \prime}, g^{\prime \prime}, g^{\prime \prime}\right\}$, after suitable renumbering, and can be summarized as $G=(1-\lambda) G_{0}+\lambda G_{1}$, where $G_{1}$ corresponds to two non-interacting atoms or mesons. The values of $g^{\prime}, g^{\prime \prime}$ and $\lambda$ are shown in Table 1 .

The variational principle, with the solution of this symmetric case as a trial function, implies that, $\min (H[G]) \leq \min \left(H\left[G_{0}\right]\right)$, so that the more asymmetric the distribution $G$, the lower the ground-state energy. For $G=(1-\lambda) G_{0}+\lambda G_{1}$, the ground-state energy $E(\lambda)$ is a convex function of $\lambda$, and, since it is maximal at $\lambda=0$, it is convexly decreasing as a function of $|\lambda|$ for both $\lambda \geq 0$ and $\lambda \leq 0$. If one compares two values of $\lambda$ with opposite sign, then it is a good guess, but not a rigorous statement, to say that the larger $|\lambda|$, the lower the energy, as $E(\lambda)$ is nearly parabolic near $\lambda=0$. The asymmetry parameter $|\lambda|$ is much larger for $\operatorname{Ps}_{2}$ than

Table 1. Strength coefficients in the potential. The parameter $\lambda$ measures the departure from the symmetric case towards the configuration with two separated atoms or mesons.

\begin{tabular}{|c|crrr|}
\hline$(1234)$ & $V(r)$ & $g^{\prime}$ & $g^{\prime \prime}$ & $\lambda$ \\
\hline $\mathrm{Ps}+\mathrm{Ps}$ & $-1 / r$ & 1 & 0 & 1 \\
$\mathrm{Ps}_{2}$ & $-1 / r$ & -1 & 1 & -2 \\
$(q \bar{q})+(q \bar{q})$ & $-1 / r, r$ & 1 & 0 & 1 \\
{$\left[(q q)_{\overline{3}}(\bar{q} \bar{q})_{3}\right]$} & $-1 / r, r$ & $1 / 2$ & $1 / 4$ & $1 / 4$ \\
{$\left[(q q)_{6}(\bar{q} \bar{q})_{\overline{6}}\right]$} & $-1 / r, r$ & $-1 / 4$ & $5 / 8$ & $-7 / 8$ \\
\hline
\end{tabular}

for Ps + Ps. This explains why the positronium molecule is bound. A tetraquark with a pure $\overline{3} 3$ color structure has its asymmetry parameter smaller than that of the threshold and has the same sign, so this toy model is rigorously unbound. For $6 \overline{6}$, the signs are different, and the magnitudes are comparable, so the analysis is more delicate. We note that $|\lambda|=2$ for $\mathrm{Ps}_{2}$ results in a fragile binding, so it is not surprising that $|\lambda|=7 / 8$ leaves $6 \overline{6}$ unbound in numerical studies.

To summarize, the tetraquark is penalized by the non-Abelian character of the color algebra, and its stability cannot rely on the asymmetries of the potential energy. It should use other asymmetries, in particular through the masses entering 
the kinetic energy, or spin effects, or mixing of $\overline{3} 3$ and $6 \overline{6}$, or the coupling to decay channels, etc.

\section{5. $Q Q \bar{Q} \bar{Q}$ states}

In the previous section, the instability of all-heavy tetraquarks with a $\overline{3} 3$ color wave function was derived from the properties of the color coefficients entering the Hamiltonian. An alternative proof is based on the so-called Hall-Post inequalities, first applied to light nuclei ${ }^{16}$, often rediscovered either in a degraded or improved form, for instance in studies related to the stability of matter, and already adapted to relate mesons and baryons ${ }^{9,17}$. The principle is rather simple. If a Hamiltonian is decomposed as a sum of Hamiltonians, $H=h^{\prime}+h^{\prime \prime}+\ldots$, then for the lowest energy,

$$
E(H) \geq E\left(h^{\prime}\right)+E\left(h^{\prime \prime}\right)+\cdots
$$

With a $\overline{3} 3$ color wave function and a quark mass set to $M=1 / 2$ for simplicity, the Hamiltonian of the all-heavy tetraquark $Q Q \bar{Q} \bar{Q}$ can be written as,

$$
H_{4}=\frac{1}{2}\left(h_{12}+h_{34}\right)+\frac{1}{4}\left(h_{13}+h_{14}+h_{23}+h_{24}\right),
$$

where $h_{i j}=\vec{p}_{i}^{2}+\vec{p}_{j}^{2}+v\left(r_{i j}\right)$ is the quarkonium Hamiltonian. By using Eq. (3) one gets,

$$
E_{\min }\left(H_{4}\right) \geq 2 E_{\min }\left(h_{13}\right)=2 E_{\min }(Q \bar{Q})
$$

that demonstrates the instability of all-heavy tetraquarks. The above reasoning on the ground state holds for a single color channel. It is observed in explicit computations than the mixing of color states does no help much ${ }^{5,18}$. The lower bound (5) can even be significantly improved if one relates Hamiltonians that are free of centerof-mass motion. More details will be given in Ref. 12 .

\section{6. $Q q \bar{Q} \bar{q}$ states}

As discussed in Sec. 3 the presence of two thresholds for $Q q \bar{Q} \bar{q}$, one of them taking benefit from the breaking of the particle identity, makes the stability of tetraquarks rather difficult unless there is a strong-enough coupling among the two threshold configurations, due, for example, to the exchange of pseudoscalar mesons. It has been explained in Ref. 19 how hadrons with a $Q q \bar{Q} \bar{q}$ flavor content, could split either into $(Q \bar{q})-(q \bar{Q})$ or $(Q \bar{Q})-(q \bar{q})$. For $Q=c$, the $(Q \bar{Q})-(q \bar{q})$ and $(Q \bar{q})-$ $(q \bar{Q})$ thresholds are not too far from degenerate, while for $Q=b$ the $(Q \bar{Q})-$ $(q \bar{q})$ threshold is much lower than the $(Q \bar{q})-(b \bar{Q})$ one. Such increase of the mass difference between the two thresholds when the mass of the heavy quark increases, is a common feature of all constituent quark models ${ }^{20}$, and can be understood from the flavor-independence of the chromoelectric part ${ }^{21}$. Thus, the possibility of finding meson-antimeson molecules, $(Q \bar{q})-(q \bar{Q})$, becomes more and more difficult when the 
mass of the heavy flavored quark increases, due to the lowering of the $(Q \bar{Q})-(q \bar{q})$ threshold. Such a $(Q \bar{q})-(q \bar{Q})$ molecule would dissociate immediately. This might explain the absence of a bottom partner of the $X(3872)$.

In the charm sector, the vicinity of the two thresholds and the strong coupling between them, give rise to a resonance in between the two channels. These arguments were the basis for the simultaneous explanation of an exotic tetraquark with two units of charm and the $X(3872)^{22}$. The vicinity of thresholds is not enough as to guarantee the existence of a bound state or a resonance: a coupling between the two thresholds is required ${ }^{23}$. The absence of such a coupling is perhaps responsible for the non-existence of a charged partner of the $X(3872)$.

\section{Acknowledgements}

This work has been partially funded by Ministerio de Economía, Industria y Competitividad and EU FEDER under FPA2016-77177 and FPA2015-69714-REDT, by Junta de Castilla y León under SA041U16, and by Generalitat Valenciana PrometeoII/2014/066.

\section{References}

1. E. Cartlidge, Physics World, Dec. $1^{\text {st }},(2017)$.

2. H. -X. Chen, W. Chen, X. Liu, and S. -L. Zhu, Phys. Rep. 639, 1 (2016).

3. R. Aaij et al. (LHCb Collaboration), Phys. Rev. Lett. 115, 072001 (2015).

4. R. Aaij et al. (LHCb Collaboration), Phys. Rev. Lett. 119, 112001 (2017).

5. J. Vijande, A. Valcarce, and N. Barnea, Phys. Rev. D 79, 074010 (2009).

6. E. A. G. Armour, J. -M. Richard, and K. Varga, Phys. Rep. 413, 1 (2004).

7. J. -M. Richard, Few Body Syst. 57, 1185 (2016).

8. J. -M. Richard, A. Valcarce, and J. Vijande, Phys. Rev. D 95, 054019 (2017).

9. J. -P. Ader, J. -M. Richard, and P. Taxil, Phys. Rev. D 25, 2370 (1982).

10. E. J. Eichten and C. Quigg, Phys. Rev. Lett. 119, 202002 (2017); A. Francis et al., Phys. Rev. Lett. 118, 142001 (2017); P. Bicudo et al., Phys. Rev. D 96, 054510 (2017).

11. M. Karliner and J. L. Rosner, Phys. Rev. Lett. 119, 202001 (2017).

12. J. Vijande et al., in preparation.

13. S. Zouzou et al., Z. Phys. C 30, 457 (1986).

14. D. Janc and M. Rosina, Few Body Syst. 35, 175 (2004).

15. J. Vijande et al., Phys. Rev. D 76, 094027 (2007).

16. R. L. Hall and H. R. Post, Proc. Phys. Soc. 90, 381 (1967).

17. J. L. Basdevant, A. Martin, and J. M. Richard, Nucl. Phys. B 343, 69 (1990).

18. J. Vijande, A. Valcarce, and J. -M. Richard, Phys. Rev. D 76, 114013 (2007).

19. T. F. Caramés, A. Valcarce, and J. Vijande, Phys. Lett. B 709, 358 (2012).

20. E. Eichten et al., Phys. Rev. Lett. 34, 369 (1975), [Erratum: ibid 36, 1276 (1976)].

21. R. A. Bertlmann and A. Martin, Nucl. Phys. B 168, 111 (1980); S. Nussinov and M. A. Lampert, Phys. Rept. 362, 193 (2002).

22. T. F. Caramés, A. Valcarce, and J. Vijande, Phys. Lett. B 699, 291 (2011).

23. J. Vijande and A. Valcarce, Phys. Lett. B 736, 325 (2014). 